\title{
The role of memory in perceiving subjective contours
}

\author{
HANS WALLACH and VIRGINIA SLAUGHTER \\ Swarthmore College, Swarthmore, Pennsylvania
}

\begin{abstract}
In the variety of subjective contours that are structured like Kanizsa's triangle, shapes consist partly of edges of elements that make up the patterns that give rise to the subjective contours and partly of the subjective contours. It was found that familiarity with the shape that fit the subjective-contour-inducing pattern in this fashion increased the likelihood that subjective contours were perceived when the "containing" pattern was shown. Six containing patterns were constructed that did not readily yield subjective contours when the fitting shapes were not familiar. It was found that these containing patterns yielded subjective contours considerably more often when their fitting shapes had been made familiar than when they had not been. This result was obtained even though all subjects were acquainted with subjective contours before the containing patterns were shown, and even though they were asked whether or not they saw subjective contours when they saw the containing patterns. In a second experiment, subjects had no such set to experience subjective contours. When two of the containing patterns that had been used in the first experiment were shown, no subjective contours were perceived. However, after subjects had been familiarized with a drawing of one of the fitting shapes, they saw that shape in the pattern that contained it and along with it the subjective contours.
\end{abstract}

In recent years, subjective contours, also called illusory contours, have been given much attention, and that is as it should be. Whenever percepts have features that do not have their counterparts in proximal stimulation, they are likely to reveal something about the perceptual processes involved. Subjective contours take many forms. They are discussed in a recent review of the large literature by Parks (1984). Our investigation deals with the kind of patterns that yield subjective contours most reliably when viewed by naive subjects, that is, by those who have not been specifically aware of subjective contours before. In these patterns, subjective contours are more or less straight connections between aligned edges in cutouts that are readily perceived as cutouts, where the subjective contours are parts of edges of simple shapes, such as the upright triangle in Figure 1. The outline of the triangle consists of real contours where it shares the edges of the cutouts in the three black disks. Where the outline of the triangle is not provided by stimulation-that is, in the straight connections between the ends of the real contours-subjective contours are seen. These parts of the triangle's outline are the perceived features that have no counterpart in stimulation. The pattern that gives rise to subjective contours-in the case of Figure 1 the three black disks and the interrupted outline of the upside-down triangle-has been called a subjective-contour-inducing array and will be referred to as a containing pattern. The figure that is composed in part of subjective contours, here

This work was supported by Grant BSN-8318772 from the National Science Foundation to Swarthmore College, Hans Wallach, principal investigator. The authors' mailing address is Department of Psychology, Swarthmore College, Swarthmore, PA 19081. the upright triangle, has been called subjective figure; its shape will be referred to as the fitting shape.

When a percept has features not given in proximal stimulation, as in the case of shapes partly composed of subjective contours, it is possible that a memorial representation contributes to them. That happens, for instance, when in the absence of binocular vision or of head movements, tridimensional shapes are perceived although only the projections of such shapes are given on a retina. Strong evidence for such effects of memories were obtained by Wallach, O'Connell, and Neisser (1953), who demonstrated that memorial representations created within an experiment could cause retinal patterns that normally evoke flat shapes to be perceived as tridimensional shapes. The authors experimented with tridimensional shapes so chosen that their projections resulted in the perception of flat shapes. In the course of that experiment, the tridimen-
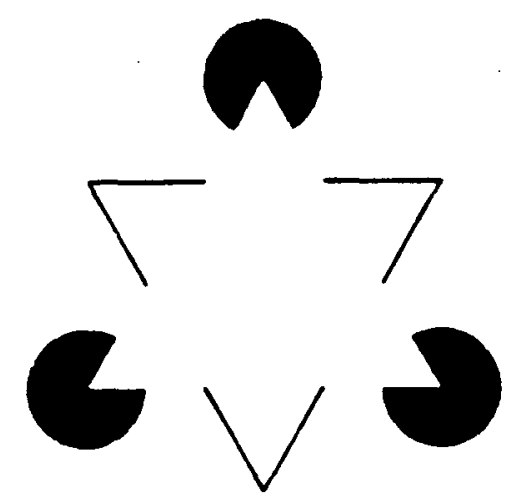

Figure 1. Kanizsa's display, yielding subjective contours. 
sional shapes were made to turn so that their projections changed form and evoked tridimensionally perceived shapes. When later stationary projections were displayed again, they were often perceived as tridimensional shapes. A stringent criterion for perceived tridimensionality was used; spontaneous reports of inversion served as evidence that now tridimensional shapes were being perceived.

Analogous experiments will be reported here that show that memorial representations contribute to the perception of shapes that, like the upright triangle in Figure 1, are partially composed of subjective contours. Instead of employing displays in which the subjective figures were simple familiar shapes, we, too, created, in the course of our experiments, the memorial representations that we believed were needed for the perception of subjective contours. Six unfamiliar shapes were designed to serve as fitting shapes, and for each of them a containing pattern was constructed (Figure 2). The containing patterns were so designed that the fitting shapes were not readily seen. But after subjects were made familiar with outline drawings of the fitting shapes (Figure 3), the fitting shapes were seen more frequently in the containing patterns, as were the subjective contours.

It was not easy to construct the containing patterns so that no subjective contours would be reported when the fitting shapes were not familiar to the subjects but would emerge from the containing patterns when the fitting shapes had been made familiar to them. This ideal outcome could be obtained only when subjects had no set to perceive subjective contours and as long as no set de- veloped in the course of the experiment; otherwise, this outcome could only be approximated. Often, subjects who have a set to perceive subjective contours look for alignments between edges of different cutouts and other enclosing pattern elements; when they find them, they perceive the connection between the aligned edges as subjective contours. If they do, they will report subjective contours, even though they may not perceive the whole subjective figure.

\section{EXPERIMENT 1}

Initially, we felt that we had to acquaint our subjects with the experience of subjective contours so that we could be sure whether or not they perceived them. The result we hoped for was that subjective contours would be perceived in those containing patterns for which the fitting shapes had been made familiar, and that they would not be perceived in patterns for which that had not been done. Both results involved hazards. For familiarization to be effective, the resulting memorial representation of the fitting shape had to become operational when the containing pattern was displayed. That would not happen when that pattern did not make contact with the memory of the shape that fitted it. Some process akin to recognition had to take place; otherwise, even when the fitting shape had been learned, subjective contours would not be perceived. On the other hand, subjects might report seeing subjective contours in containing patterns for which the fitting shapes had not been made familiar, because they had

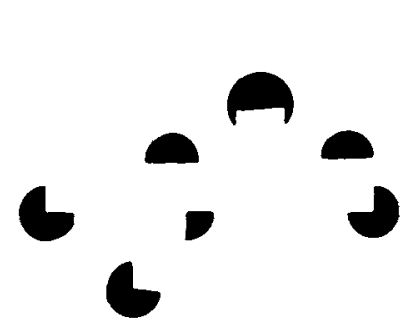

A

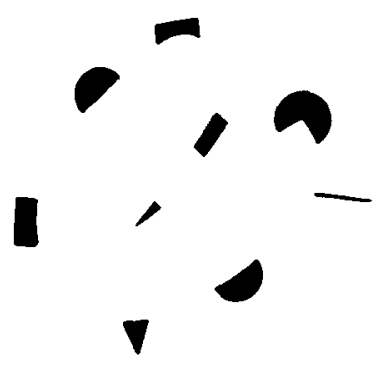

D

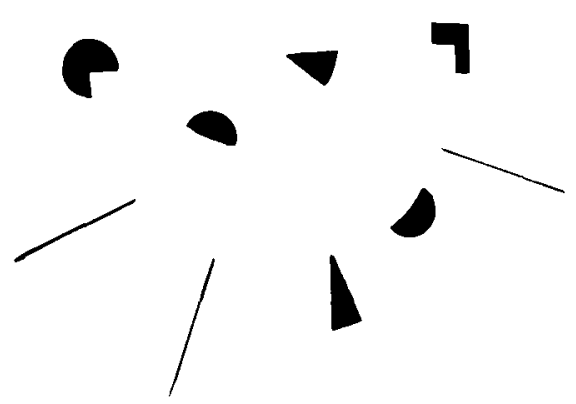

B

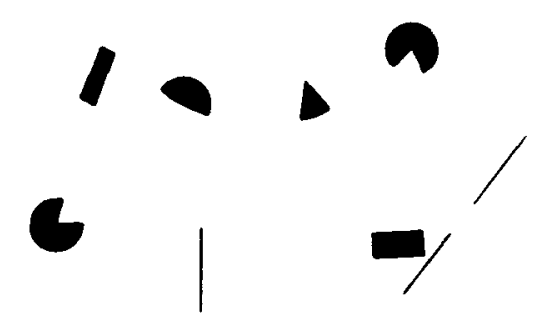

E
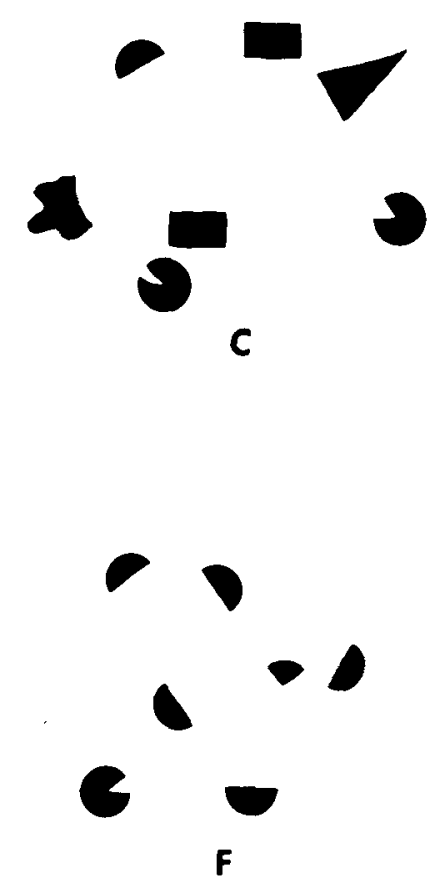

Figure 2. Six containing patterns employed in Experiment 1. 


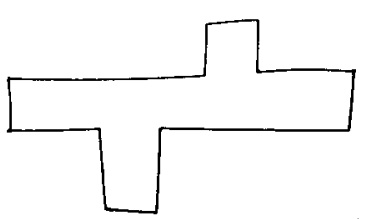

A

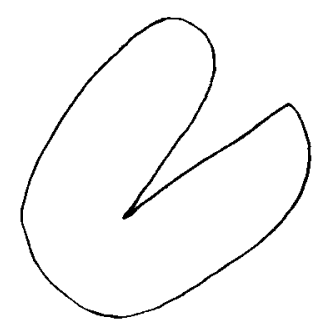

D

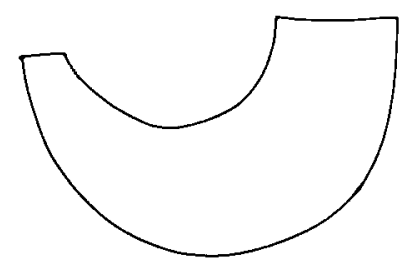

B

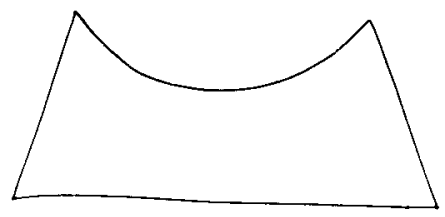

E

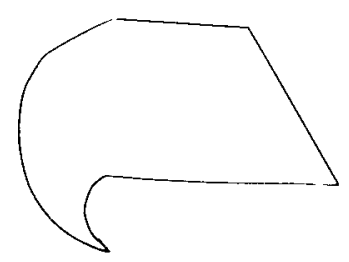

C

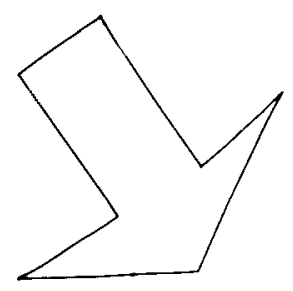

$F$

Figure 3. Drawings of the fitting shapes of the six containing patterns.

looked for alignments between edges of the cutouts and other edges of pattern elements. The two hazards diminished the difference between the results for the containing pattern for which the fitting shapes had been made familiar (experimental condition) and the results for the containing patterns for which this had not been done (control condition).

The experiment consisted of three steps. First, three of the six fitting shapes were made familiar to the subject. Then the subject was made acquainted with subjective contours. Finally, all six containing patterns were shown to the subject, one after the other, and the subject had to report, in each case, whether he or she saw subjective contours.

\section{Method}

Subjects. Fifty-four undergraduates were paid for serving as subjects.

Equipment. In addition to the six containing patterns shown in Figure 2 and the six outline drawings of the fitting shapes (Figure 3), there were four patterns that resembled the containing patterns but could not yield subjective contours because none of the edges of the pattern elements were aligned. They will be called $\mathbf{N}$ patterns. Finally, there was the containing pattern that was used to make the subjects familiar with the experience of subjective contours (Figure 4). It was chosen for this purpose because its fitting shape was not immediately seen by naive subjects but yielded impressive subjective contours when it was. All these figures were drawn on $20 \times 20 \mathrm{~cm}$ cards and were observed from a distance of $152 \mathrm{~cm}$.

Procedure. All subjects were first made familiar with three of the outline drawings of fitting shapes (Figure 3). First they learned to associate each of the three drawings with its letter name, and then they had to draw each from memory and supply its name. Oddnumbered subjects learned in this fashion the fitting shapes $A, B$, and $C$; even-numbered subjects learned the shapes D, E, and F.
Next, all subjects were shown Figure 4. They were asked what they saw and were made to look at the pattern until they reported seeing a cross. Then the experimenter pointed to an area where the subject presumably saw a subjective contour. Calling it an "illusory contour," the experimenter said: "Even though you may feel that you are perceiving a line here, surrounding and defining the cross, there is not any such line. Such an illusory contour seems to be a line enclosing a whole cohesive figure." The experimenter then explained that the subject would be shown some charts similar to that of the cross, and that some would contain illusory contours while others would not. The subject was instructed to say "yes" if he or she saw an illusory contour and "no" if he or she did not.

During the subsequent test presentation, each chart was shown for approximately $4 \mathrm{sec}$, sufficient time for every subject to arrive at a decision. All six containing patterns were presented, the three

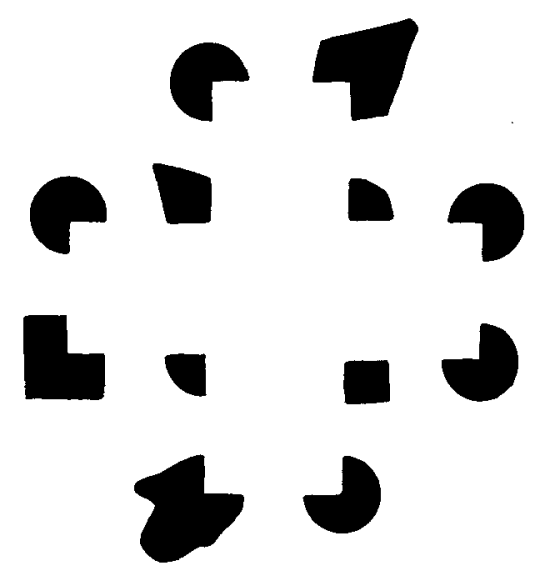

Figure 4. Containing pattern employed to acquaint subjects with subjective contours. 
for which the fitting shapes had been made familiar and the three for which they had not. In addition to the six critical patterns, all subjects were shown the $\mathrm{N}$ patterns. All subjects saw the six containing patterns and the four $\mathbf{N}$ patterns in the same order: $\mathrm{N} 1, \mathrm{~N} 2$, A, D, N3, B, E, N4, C, F. This was our way of controlling for an order effect. A particular pattern whose fitting shape had been made familiar to one group of subjects occupied exactly the same position in the presentation sequence when, for the other group, its fitting shape was unfamiliar. Although randomization of the order of the containing patterns would have provided information about an order effect, the fixed-order procedure was used because it provided optimal conditions for dealing with the effect of familiarity.

\section{Results}

Table 1 lists the number of subjects who reported seeing a subjective contour in the case of each of the containing patterns when its fitting shape was familiar (first row) or when it was not (second row) and in the case of the four $\mathrm{N}$ patterns combined (third row). The 162 containing patterns whose fitting shapes had been made familiar elicited a total of 115 , or $71.0 \%$, "yes" responses. The 162 containing patterns whose fitting shapes were unfamiliar elicited 66 , or $40.7 \%$, "yes" responses. Altogether, the 216 presentations of $\mathrm{N}$ patterns elicited 23 "yes" responses, providing an estimate of random "yes" responses of $10.6 \%$. This rate of random "yes" responses cannot account for a rate of $40.7 \%$ of "yes", responses that were given in the control condition; a high proportion of these cases were caused by a set. However, the difference between the 66 cases in which the unfamiliar fitting shapes (control condition) produced "yes" responses and the 115 cases in which the familiar fitting shapes (experimental condition) produced "yes" responses can be ascribed to the familiarity of the fitting shapes. If one takes into account that nothing was done to facilitate containing patterns' making contact with memorial representations of the fitting shapes, the rate of $71.0 \%$ "yes" responses in the experimental condition seems quite satisfactory.

An analysis of subjects' "yes" responses in the experimental and control conditions, using Wilcoxon's matched-pairs signed-ranks test, yielded $z=-4.34$ and $p=.00006$.

This result encouraged us to try to experiment with subjects who had not been made acquainted with subjective contours in the course of the experiment. With such subjects, the frequency with which subjective contours would be reported in the control condition should be greatly diminished. In this experiment, a subject was presented with only one containing pattern whose fitting shape was familiar and was familiarized with only two fitting shapes.

Table 1

Number of Subjects Who Reported Seeing Subjective Contours When Viewing Containing Patterns Representing Familiar or Unfamiliar Shapes and When Viewing $\mathbf{N}$ Patterns

\begin{tabular}{lrrrrrrr}
\hline Pattern & \multicolumn{7}{c}{ Patterns } \\
\cline { 2 - 7 } Category & A & B & C & D & E & F & Totals \\
\hline Familiar & 26 & 16 & 14 & 14 & 21 & 24 & 115 \\
Unfamiliar & 16 & 7 & 14 & 5 & 14 & 10 & 66 \\
N pattern & & & & & & & 23 \\
\hline
\end{tabular}

\section{EXPERIMENT 2}

\section{Method}

Subjects. Twenty-six undergraduates were paid for serving as subjects.

Equipment. The containing patterns A and $\mathrm{F}$ were selected from those of Experiment 1 because they most resembled conventional subjective contour patterns. They served as critical items, that is, the outline drawings of their fitting shapes were also used. The outline drawing of shape E served as a filler.

Procedure. First, every subject was shown the containing patterns $A$ and $F$ and, in each case, asked to describe it (first presentation). The order in which these patterns were shown was varied. If, in either one of the two cases, the subject reported seeing the fitting shape, for example, an arrow or a white figure on the black circles, he or she was disqualified. There were 4 so-disqualified subjects. Thus, 22 subjects completed the experiment.

Then the subject was made familiar with the outline drawing of either fitting shape A or fitting shape $F$ and with the outline drawing $E$. When the subject felt ready, after being shown the two drawings alternately several times, he or she drew the shapes and gave their names. When it came to the test, 11 subjects were familiar with the fitting shape $A$ but not with fitting shape $F$ and 11 were familiar with the fitting shape $F$ but not the fitting shape $A$.

In the test, all subjects were shown both containing patterns, A and $F$, with one of the patterns always serving as control. As before, the subject was asked to describe what he or she saw. A subject was always first presented with the containing pattern whose fitting shape had not been made familiar. (Because it turned out that the fitting shape was never seen in that containing pattern, subjects had still not seen subjective contours during the experiment when the critical containing pattern was presented.)

\section{Results}

When the containing pattern whose fitting shape had not been made familiar was shown, all subjects gave a description similar to the one they had given at the first presentation; the fitting shapes never occurred in these descriptions. When next they were shown the other, critical containing pattern, 15 subjects reported seeing the fitting shape, 6 of them immediately and 9 after looking at the pattern for a brief time. The other 7 subjects saw the fitting shape after they had been asked: "Can you see one of the figures you have learned before?" This caused them to see the fitting shape. (This question could not be asked in Experiment 1, and this probably accounts in large part for the 162 minus 115 cases in which no subjective contours were reported in the experimental conditions.)

After the subject had seen the fitting shape, either spontaneously or after being prompted, we made sure that he or she also saw its subjective contour. Twelve of the subjects were asked to follow the edge of the shape they saw with a finger. They all traced the outline correctly and answered positively to the question: "Do you see it as if there were a line around the figure, even in the white spaces?" The other 10 subjects were then shown a compelling subjective contour pattern (Figure 4), and the experimenter said: "This is what we call an illusory contour; there seems to be an outlined shape even though there isn't actually a line surrounding it. Did you see the same thing in the figure I just showed you?" All 10 subjects answered "yes" to this question. 
The probability that all 22 subjects saw the subjective figure where the fitting shape had been made familiar and none where that was not the case is $2.4 \times 10^{-7}$.

This experiment demonstrated that only subjects familiar with the fitting shape reported seeing it in the containing pattern, that these subjects did so either spontaneously (15) or when associative access to the memorial representation was provided (7), and that all subjects who saw the fitting shape in the containing pattern also saw subjective contours.

\section{SUMMARY AND DISCUSSION}

An important condition for the perception of subjective contours is familiarity with the fitting shape and a coming into play of the memories that represent the familiarity. In fact, it may well be the only condition that, in the absence of a perceptual set, results in a perception of subjective contours, unless a stereoscopic depth effect provides the fitting shape (see below). In Experiment 1, in which all subjects were made acquainted with subjective contours and in which instructions caused a set to look for them, subjective contours were still 1.74 times as frequently perceived when the fitting shapes had been made familiar than when they had not been. When, in Experiment 2 , subjects were free of a set to look for subjective contours and remained so until the critical exposure, no subjective contours were perceived unless the subject had been made familiar with the fitting shape. When the memorial representation of the fitting shape came into play, subjective contours were perceived in every case.

In the usual demonstrations of subjective contours, the fitting shapes are simple geometric forms that are highly familiar, such as equilateral triangles and squares. Sixty or $90^{\circ}$ cutouts in elements of the containing patterns serve to make contact with the memorial representations of these simple shapes. In the most reliable demonstration, that using Kanizsa's triangle (Figure 1), the relevant memory, that of a triangle, is brought into play by the outline triangle that is part of the display.

That the emergence of subjective contours may be favored by a perceptual set has occasionally been noted, but formal experimental evidence has been presented only recently. Coren, Porac, and Theodor (1986) published two experiments that demonstrated the effectiveness of set. Their Experiment 2 demonstrated a general set produced by prior exposure to subjective-contour-inducing patterns. This is comparable to the general set that operated in our Experiment 1, in which subjects were first made acquainted with a subjective-contour-producing pattern and were under instruction to report subjective contours when they saw them. Coren et al.'s (1986) Experiment 1 is more relevant to this discussion. Their test figure consisted of four thin lines forming an upright cross with a gap where the crossing point would have been. Upon suggestion that this gap may be seen as a white form and that subjects should draw a line around the form if they saw it, 90 out of 161 subjects did so. This set was thus $56 \%$ effective. However, the important finding was that naming the shape of the white form was also partially effective. When the white form was referred to as "figure," the result was 20 drawings of a circular outline, 2 of a square, and 4 amorphous outlines by 51 subjects; when it was referred to as a circle, the result was 39 circular outlines from the 58 subjects; and when it was called a square, the result was 5 circular and 20 square outlines from 52 subjects.

Coren et al. (1986) found these results "consistent with cognitive explanations of subjective-contour formation" and compatible with several theoretical positions: Subjective contours may be "a form of problem solving, ... a form of Gestalt closure," or arise from a "reorganization of the configuration on the basis of implicit depth cues"' (p. 332). Our experiments, on the other hand, support the view that subjective figures are manifestations of memorial representations coming into play and being part of the form process. Coren et al.'s finding that instructions containing the words "circle" and "square" often influenced the shape of the subjective figure if one was seen is in agreement with our conclusion. Such instructions may bring memorial representations of these shapes into play and thereby determine the shapes of the subjective figures.

That subjective contours occur when information carried by stimulation is incomplete and when memory supplies the missing pieces shows that their perception is similar to that of a tridimensional form that, in the absence of depth cues from stimulation, also results from the operation of memorial representations. But there is a difference. In the perception of tridimensional form, memorial representation merely gives shape to what is given on the retina. In the case of subjective contours, memorial representations add material features to perception. This suggests imagery. But we hesitate to regard the subjective figure with its completed contours as an image fitted into the containing pattern; mental images are not known to fit themselves into percepts.

There are two conditions under which subjective contours are seen that are not completions of edges in stationary patterns. They are discussed here because they fit with our findings in various ways. One of the conditions involves dot-matrix stereograms, as designed by Julesz (1960), that give rise to a segregation of a part of the dot pattern. The segregation may be achieved by dichoptic presentation of the stereograms causing stereoscopic depth to be perceived, with the inner region appearing in front of the outer region. Before Kanizsa's subjective contours became widely known, White (1962) noted that the inner region seems to have an edge that "lies between the rows and columns of dots.... This is a rare instance of visual contour-formation in the absence of a brightness gradient in the field" (p. 411). Later, Lawson and Gulick (1967) produced a whole subjective figure stereoscopically by omitting the dot pattern in the inner region. The other way to bring about such segregation and to cause such subjective contours to form is by 


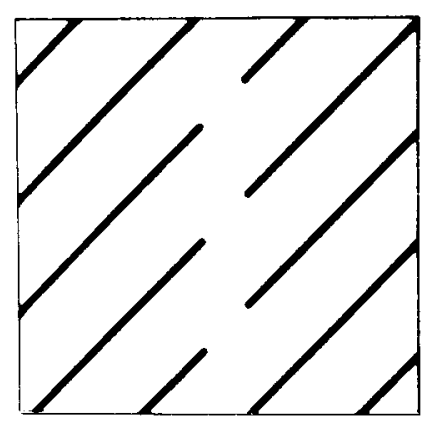

Figure 5. Moving-line display with vertical gap in line pattern.

alternately projecting the left-eye and the right-eye matrix into the same area in rapid sequence. The inner region will then appear to move horizontally back and forth slightly in front of the outer region (White, 1962). The finding that in both cases segregation is an antecedent of the perception of subjective contours agrees with the notion that is implicit in our findings: Subjective contours result when shapes are perceived whose edges are not given or are only partly given in stimulation. In Kanizsatype displays, memorial representations furnish the shapes, and in Julesz-type stereograms, stereoscopic depth or White's stroboscopic motion result in segregation which provides the shapes.

The other condition for subjective contours involves moving line patterns. When a pattern of parallel and regularly spaced oblique lines moves behind a screen and is seen through an aperture in the screen, even though the objective direction of the motion of the pattern is not given, the perceived motion has a definitive direction, and that has interesting consequences (Wallach, 1935; see also Wallach, 1976, chap. IX, 1). When, for instance, such line-pattern motion is given in a square aperture, the perceived motion is parallel to the aperture edges. Since there are two pairs of aperture edges, two motion directions are possible, one vertical and the other horizontal. Per-

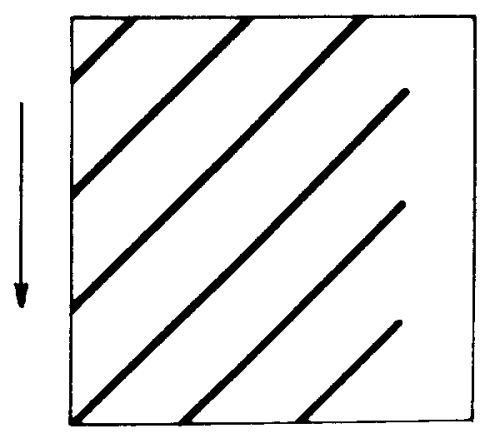

Figure 6. Moving-line display with lines ending on the right side, inside the frame. ception that results from such ambiguous stimulation conditions as a rule yields to perceptual satiation; the two possible versions are experienced in alternation. The line motion is no exception: the pattern appears to move alternately vertically and horizontally. Satiation has a strong effect. Motion direction changes even when cues for the objective motion are present. When a line pattern with a vertical gap (Figure 5) moves vertically downward, vertical motion is seen for much longer than it is in the ambiguous arrangement, because the visible ends of the lines serve as cues for the objective motion direction. When eventually the lines are seen to move horizontally, the gap is perceived as a vertical white strip with subjective contours on either side where the lines objectively have their ends. These ends are no longer seen; the line pattern seems to be whole and to be moving behind the strip. This case fits our explanation of the subjective contours that are structured like Kanizsa's triangle. In this case, too, memory supplies the shape that fits into the pattern, into the gap between the end of the lines, the memory of objects that conceal part of a pattern. It appears that the strip results from two process steps, the development of horizontal motion of the pattern and a recall step that causes perception of the strip. Wallach $(1935,1976)$ investigated a similar arrangement, in which, instead of a gap in the line pattern, the pattern ended laterally. With this arrangement (Figure 6), many subjects saw the pattern move horizontally several times before they "saw clearly" what happened at the right side of the pattern. where the lines seemed to disappear under a vertical strip. Prior to the formation of the strip, the line seemed to vanish unaccountably. The two process steps often took place at different times.

\section{REFERENCES}

Coren, S., Porac, C., \& Theodor, L. H. (1986). The effects of perceptual set on the shape and apparent depth of subjective contours. Perception \& Psychophysics, 39, 327-333.

JuLESz, B. (1960). Binocular depth perception of computer-generated patterns. Bell System Technical Journal, 39, 1125-1162.

LAwson, R. B., \& Gulick, W. L. (1967). Stereopsis and anomalous contour. Vision Research, 7, 271-297.

PARKs, T. E. (1984). Illusory figures: A (mostly) atheoretical review. Psychological Bulletin, 95, 282-300.

WALLACH, H. (1935). Über visuell wahrgenommene Bewegungsrichtung. Psychologische Forschung, 20, 325-380.

WALLACH, H. (1976). On perception. New York: Quadrangle/The New York Times Book Co.

Wallach, H., O'ConNell, D. N., \& Neisser, U. (1953). The memory effect of visual perception of three-dimensional form. Journal of Experimental Psychology, 45, 360-368.

WHITE, B. W. (1962). Stimulus-conditions affecting a recently discovered stereoscopic effect. American Journal of Psychology, 75, 411-420.

(Manuscript received March 2, 1987; revision accepted for publication August 24, 1987.) 